\title{
ASYMPTOTIC VALUES OF HOLOMORPHIC FUNCTIONS OF IRREGULAR GROWTH
}

\author{
BY ALFRED GRAY AND S. M. SHAH ${ }^{1}$
}

Communicated by Maurice Heins, May 3, 1965

Let $f(z)=\sum_{n=0}^{\infty} a_{n} z^{n}$ be holomorphic with radius of convergence $R(0<R \leqq \infty)$, and let $\mu(r)$ denote the maximum term and $\nu(r)$ the central index of $f(z)$. By definition, for $r>0, \mu(r)=\max \left\{\left|a_{n}\right| r^{n} \mid n\right.$ $=0,1,2, \cdots\}$ and so $\mu(r)=\left|a_{\nu(r)}\right| r^{\nu(r)}$. We shall assume that $\mu(r) \rightarrow \infty$ as $r \rightarrow R$ and $f(z)$ is not a polynomial. In this note we give a technique for comparing $f(z)$ with its maximum term which shows that, for certain functions $f(z)$ which are of very slow growth, or whose power series have wide gaps, $f(z)$ has no finite asymptotic values. Our result is to be compared with Wiman's theorem [1, Chapter 3], [5]: If $f(z)$ is an entire function of order $\rho<\frac{1}{2}$ then $f(z)$ has no finite asymptotic values. However, the class of functions for which we show the nonexistence of finite asymptotic values is different from that of Wiman; in particular we allow the functions to have a finite radius of convergence.

Let $z=r e^{i \theta}$ and define

$$
\mu\left(r e^{i \theta}\right)=\mu(r) e^{i \nu(r) \theta}
$$

for $r>0$ and $0 \leqq \theta<2 \pi$. Then $\mu(z)$ is a complex extension of $\mu(r)$; it is piecewise continuous, but has discontinuities where $\nu(|z|)$ is discontinuous.

Let $\gamma(t)$ be a (continuous) receding curve such that $|\gamma(t)| \rightarrow R$ as $t \rightarrow \infty$. Then $\gamma(t)$ is an asymptotic path of $f(z)$ if as $t \rightarrow \infty, f(\gamma(t))$ tends to a limit $\omega$, called an asymptotic value; analogously with this definition we shall call $\gamma(t)$ a $\mu$-asymptotic path if $f(\gamma(t)) / \mu(\gamma(t))$ tends to a limit $\omega$ as $t \rightarrow \infty$, and we say that $\omega$ is a $\mu$-asymptotic value. For example, $e^{z}$ has $\mu$-asymptotic value $\infty$ along the positive real axis, but has $\mu$ asymptotic value 0 along any path to $\infty$ in any angle which excludes the positive real axis. The following theorem is obvious, since $\mu(r)$ $\rightarrow \infty$.

THEOREM 1. If $\gamma(t)$ is an asymptotic path of $f(z)$ with finite asymptotic value, then $\gamma(t)$ is a $\mu$-asymptotic path of $f(z)$ with $\mu$-asymptotic value 0 .

Next we investigate some situations in which $f(z)$ has no $\mu$-asymp-

\footnotetext{
1 The work of this author was supported by NSF grant GP-4311.
} 
totic values. Without loss of generality assume $a_{0} \neq 0$. Let $\{\rho(n)\}$ be the sequence of jump points of $\nu(r)$, counting multiplicity. Since $\nu(r) \rightarrow \infty$ as $r \rightarrow R, \rho(n) \rightarrow R$ as $n \rightarrow \infty$. We denote by $\left\{n_{k}\right\}$ the range of $\nu(r)$, so that $\nu\left(\rho\left(n_{k}\right)\right)=n_{k}$, and we define $n_{0}=0$. Then $0<\rho\left(n_{k}\right)$ $<\rho\left(n_{k}+1\right)=\cdots=\rho\left(n_{k+1}\right)<\cdots$.

Explicitly

$$
\rho\left(n_{k}\right)=\left|\frac{a_{n_{k-1}}}{a_{n_{k}}}\right|^{1 /\left(n_{\left.k-n_{k}-1\right)}\right.} .
$$

We define

$$
\begin{aligned}
& L=\limsup _{k \rightarrow \infty} \frac{\rho\left(n_{k+1}\right)}{\rho\left(n_{k}\right)}, \\
& S=\limsup _{k \rightarrow \infty}\left(n_{k+1}-n_{k}\right), \\
& \left.\begin{array}{l}
\Phi \\
\phi
\end{array}\right\}=\lim _{k \rightarrow \infty} \sup \left(n_{k+1}-n_{k}\right) \log \left\{\frac{\rho\left(n_{k+1}\right)}{\rho\left(n_{k}\right)}\right\}, \\
& \left.\begin{array}{c}
\Xi \\
\xi
\end{array}\right\}=\lim _{k \rightarrow \infty} \sup _{\inf }\left(n_{k}-n_{k-1}\right) \log \left\{\frac{\rho\left(n_{k+1}\right)}{\rho\left(n_{k}\right)}\right\} \text {. }
\end{aligned}
$$

The proofs of the following theorems are given in [2], [3], and [4].

THEOREM 2. If $L>1$ and $S<\infty$, then $f(z)$ has no $\mu$-asymptotic values. (The hypothesis $L>1$ implies that $f(z)$ is a transcendental entire function.)

THEOREM 3. Suppose that $f(z)$ has the form $f(z)=\sum_{k=0}^{\infty} a_{n_{k}} z^{n_{k}}$ where $\left\{n_{k}\right\}$ is the range of $\nu(r)$. If any of the conditions (1)-(4) hold, then $f(z)$ has no $\mu$-asymptotic values.

$$
\begin{aligned}
\phi & =\Xi=\infty, \xi>0 . \\
\xi & =\Phi=\infty, \phi>0 . \\
R & =\infty, \quad \sum_{k=1}^{\infty} \frac{1}{n_{k+1}-n_{k}}<\infty, \quad \phi=\infty, \xi>0 . \\
R & =\infty, \quad \sum_{k=1}^{\infty} \frac{1}{n_{k+1}-n_{k}}<\infty, \quad \xi=\infty, \phi>0 .
\end{aligned}
$$

TheOREM 4. Suppose that $f(z)$ has the form

$$
f(z)=\epsilon(0)+\sum_{k=1}^{\infty} \frac{\epsilon(k) z^{n_{k}}}{\rho(1) \cdots \rho\left(n_{k}\right)} .
$$


Assume $|\epsilon(k)|=1$ and $\epsilon(k)$ has period $h$ where $h$ is a positive integer. If $0<\phi=\Phi<\infty$ and $0<\xi=\Xi<\infty$, then $f(z)$ has no $\mu$-asymptotic values.

Theorem 1, combined with Theorems 2, 3 and 4, yields

TheOREM 5. If the hypotheses of Theorems 2, 3, or 4 are satisfied, then $f(z)$ has no finite asymptotic values.

EXAMPLES. Each of the following functions has no finite asymptotic values:

$$
\begin{array}{cc}
\sum_{k=0}^{\infty} \frac{z^{k} \exp i \alpha_{k}}{\lambda^{(1 / 2) k(k+1)}}, & \sum_{k=0}^{\infty} \frac{z^{p k-1} \exp i \alpha_{k}}{\Gamma\left(\alpha p^{k}+1\right)}, \\
\sum_{k=0}^{\infty} \frac{z^{p^{k}} \exp i \alpha_{k}}{\{(k+\lambda) \log p\}^{p^{k}}}, & 1+\sum_{k=1}^{\infty} \frac{z^{k^{p}}}{\Gamma\left(\alpha k^{p}+1\right)}, \\
1+\sum_{k=1}^{\infty} \frac{z^{k^{q}}}{\{(k+\lambda) \log q\}^{q}}, & \text { and } \sum_{k=1}^{\infty} \exp \left(\frac{p^{k \beta}}{\beta}\right) z^{p k-1},
\end{array}
$$

where $\lambda>1, \alpha>0,0<\beta<1,0 \leqq \alpha_{k}<2 \pi, p$ is an integer greater than 1 , and $q$ is an integer greater than 2 .

\section{REFERENCES}

1. R. P. Boas, Entire functions, Academic Press, New York, 1954.

2. Alfred Gray and S. M. Shah, Holomorphic functions with gap power series, Math Z. 86 (1965), 375-394.

3. - Asymptotic values of a holomorphic function with respect to its maximum term, Pacific J. Math. (to appear).

4. - Holomorphic functions with gap power series. II, J. Math. Anal. Appl. (to appear).

5. G. Valiron, Integral functions, Chelsea, New York, 1949.

University of California, Berkeley aNd

UNIVERSITY OF KANSAS 